\title{
Mechanical Properties of Polyester Sandwich Composites Wood Flour Reinforced Fiberglass
}

\author{
Azwar Yunus ${ }^{1}$, Saifuddin $^{1}$, and Adi Saputra Ismi ${ }^{1}$ \\ \{azwaryunus@pnl.ac.id\} \\ Department of Mechanical Engineering, Politeknik Negeri Lhokseumawe, Aceh, Indonesia
}

\begin{abstract}
The use of wood flour as filler in thermoset polyester composites has some advantageous since it is cheap and abundant, mild, not abrasive, and environmentally friendly. In this study, these advantages are in depth explored in order to produce an innovative sandwich composite material that meets the requirements of engineering construction material for various purposes. Study of mechanical properties is focused on the preparatory process of the wood flour till the formation of a composite sandwich. The effect of pre-treatment on wood flour, size and dimensions as well as the reinforcing process using fiberglass to form the sandwich construction, then it studied experimentally. The specimens manufacturing by casting in open mould and testing are using the ASTM C 1341-06. The results showed that the treatment process at the wood flour and the addition of coupling agent MAH did not have a positive influence on the mechanical properties of wood flour polyester composite. The soft wood filler with the medium to coarse size of filler and the composition within $\pm 60 \%$ polyester resin has the best bending strength. Therefore, the size factor, dimensions and type of wood flour as filler has the influence on the mechanical properties, whereas the filler size of 2-3 mm eligible to be used economically and technically. While wood flour composite reinforcement with fiberglass as the sandwich type has been able to increase its bending strength up to $\pm 45 \%$ fibres and making this material allows it to be used as construction material. The types of fiberglass also have an effect, in which the type of woven fiberglass has a reinforcing effect that up to $45 \%$ better than fiberglass type CSM, but use 2 layers of fibre CSM, gains almost the same as the first layer Roving fiberglass type.
\end{abstract}

Keywords: Wood flour filler, Polyester composite, Mechanical properties, Fiberglass, and Sandwich composite.

\section{Introduction}

Throughout the human history, wood has been used widely in almost all facets of human life both for structural or non-structural purposes such as housing, furnishings, bridge structures, vehicles, boats, ships, railroads, paper materials, etc. As a result, wood always sought to meet the needs of various sectors. Therefore, due to an imbalance of demand and supply, wood with the good quality become expensive and inadequate [1]. Consequently, engineers then seek for some alternatives material that design base on the wood into the alternative solutions and constantly being developed to meet the increasing demands. The wood composite is one of the products that are made, wherein the wood in various forms (powder, wood fiber, sheet) at the join using an adhesive to form a new material in the form such as particle board, fiberboard, plywood, hardboard, plywood, thermoplastic and thermoset 
of wood composite [2]. The use of various types of material tailored to the needs of the product to be made that the mechanical properties of wood composite must be meet to the technical requirements of the product.

When compared to the fiber composites, the wood flour composite tend to be more brittle because the wood flour is lack of capability of absorbing energy as well as that carried by the fiber composite. However the wood flour that has dimension and size of longer form look like the short fibers have the energy absorption better thus, it is more resilient than the wood flour composite square-shaped [3]. Proper to create a composite sandwich between the wood flour composite (core) and a thin layer of fiberglass composites on both sides as a skin can improve the mechanical properties [4]. The use of wood flour as filler will also improve the efficiency of wood flour utilization, which has not been fully utilized, and many were wasted in vain. In Aceh Province, the potencies of wood flour as filler are huge, producing by the wood processing of home industry. Filler type of wood dust is very economical in its use, since it is easy to obtain and inexpensive, nature also can prevent the occurrence of slippage in the resin and can be fastened properly by resin [5].

\section{Methods}

\subsection{Materials and Equipment}

The materials used in this research are unsaturated polyester resin BQTN 157-EX, hardener peroxide. The fiberglass type of chopped strand-mat (CSM) $300 \mathrm{gr} / \mathrm{m}^{2}$ and woven Roving $\pm 550 \mathrm{gr} / \mathrm{m}^{2}$. Moreover, the equipment used is the universal testing machine (UTM) for testing the mechanical properties, optical microscopy for fractography and high-resolution digital camera for macro photos.; molding, fiberglass roller and brush, micrometer, fernier caliper, jigsaw and milling machine for the formation of the test specimen.

\subsection{Research Procedures}

The research was designed in several stages: (1) preparing an open mold and (2) preparing the wood flour in various sizes (fine, medium, coarse to very coarse) through screening, (3) provide treatment borax alkaline and borax on the wood flour (4) preparing the unsaturated polyester resin, hardener Mekpo and fiberglass, (5) mixing the resin with wood flour with a volume fraction of 50:50 \% volume and stir for \pm 3 minutes, and then allowed to stand for \pm 3 minutes so that air bubbles disappear, then mixing with hardener Mekpo $\pm 1 \% \mathrm{wt}$ of the resin and stirred slowly for \pm 1 minute, (6) the molding process to produce a wood flour polyester composite specimen; that is made through the stages of making one layer of composite fiberglass $( \pm 1 \mathrm{~mm}$ thick) on the bottom of the mold, then a mixture of polyester resin and wood powder and hardener is poured into the top layer of the composite polyester fiberglass with a thickness of $8 \mathrm{~mm}$; then on the upper surface of the first layer of the composite made of polyester fiberglass with a thickness of $1 \mathrm{~mm}$. (8) the process of hardening (curing time), which takes between 10-15 hours, until the mold can be opened. The process sequence is repeated by varying the size and type of wood flour and the type of fiberglass.

Furthermore, 3-point bending test specimens were made through the process of cutting and sanding/polishing the specimen base on the standard ASTM C 1341-06 with the specimen 
size refers to the $\mathrm{L} / \mathrm{D}=16 / 1$; thickness $(\mathrm{d})=10 \mathrm{~mm}$, support length $(\mathrm{L})=16 \mathrm{~mm}$, the length of specimenl $=18 \mathrm{~mm}$ and specimen width $=20 \mathrm{~mm}$.

\section{Result and Discussion}

\subsection{Effect of the alkaline and the borax treatment of the wood flour filler on the bending strength.}

The alkaline treatment is intended to improve the surface of the wood flour for improving the interface bonding with polyester resins and the borax treatment is done to preserve the wood flour. Figure 1 indicates the effect of these two types of treatment on the bending strength of wood flour composite.

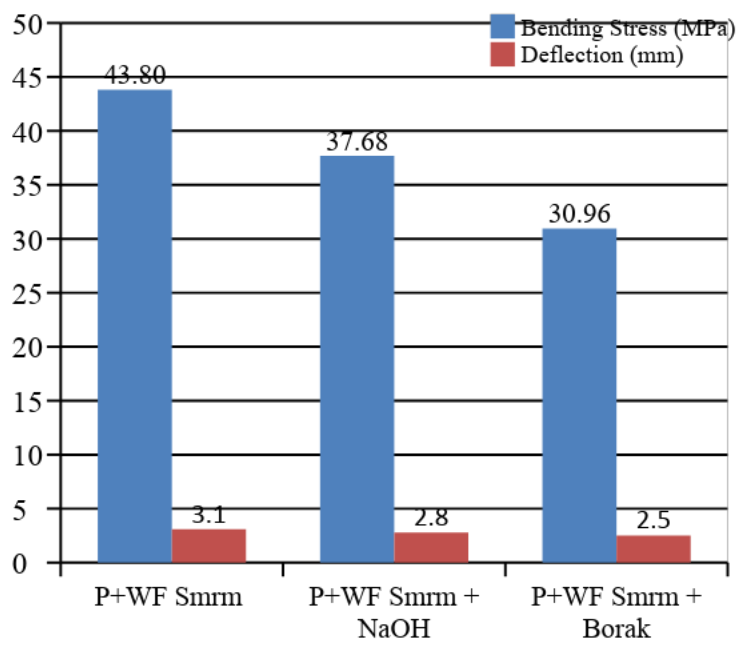

Figure 1. Effect of alkaline treatment and borax to the bending strength of polyester composite wood powder Seumaram (smrm)

Figure 1 illustrates that the wood flour of Seumaram polyester composite that do not undergo the process of alkaline or borax treatment have better bending strength, so it can be seen that the experimental treatment on wood flour filler no positive effect for increasing the strength of the composite material. This is related to the condition of the wood flour used is the wood flour option that has been dried naturally so that alkaline treatment to remove lignin and cellulose does not give a positive effect, even possible for damaging the structure of the wood flour filler that contributed to reduce the strength of material as indicated in Figure 1. Economically, this is very beneficial, given the abundant and less valuable wood flour can be directly processed into wood flour composite materials without having to go through a chemical process that is expensive, but it possible to process as naturally through drying. 


\subsection{Effect of size and dimension of wood flour on the bending strength of wood flour composites}

The calculation of bending strength of wood flour polyester composite material used wood flour in the various sizes and a square dimension is shown in Figure 2. The size and type of wood flour have an effect on the strength of the wood flour polyester composite material. The types of wood flour that studied were the kind Damasui, Jeumpa as soft wood species and hardwood species as Cengai made with the composition of 50:50. The wood flour of medium size $(1-2 \mathrm{~mm})$ and fine powder has the most strength compared to coarse wood flour. The strength of wood flour polyester composite that use the rough of wood flour also has a pretty good strength that can be improved further through the sandwich process with the fiberglass. So that the manufacture of the wood flour polyester composite or sandwich composite with fiberglass can directly use the wood flour the wood processing home industry, without having to go through a complex treatment process such as smoothing that required an additional cost.

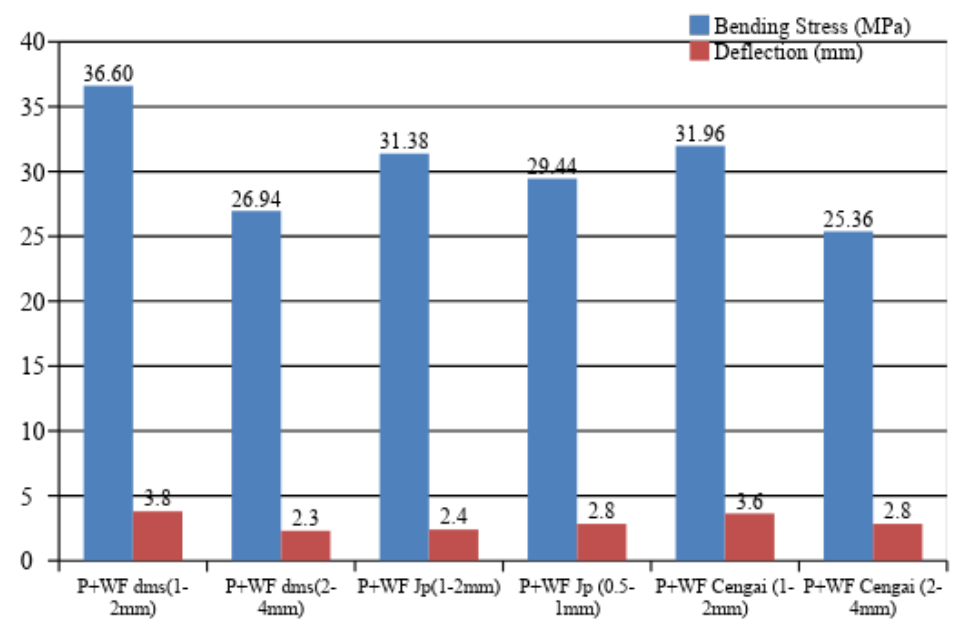

Figure 2. Effect of the size of the wood flour particles (WF) Damasui (DMS), Jeumpa(Jp) and Cengai on the bending strength of the wood flour polyester composite.

The size and dimensions of wood flour obtained from wood processing home industry in several of size and dimension. There are 4 types of the size and dimensions of wood flour in the investigation that is moderate/medium $(1-2 \mathrm{~mm})$, coarse $(2-3 \mathrm{~mm})$ and very coarse $(3-5$ $\mathrm{mm})$ and the length dimension as shown in Figure 3.
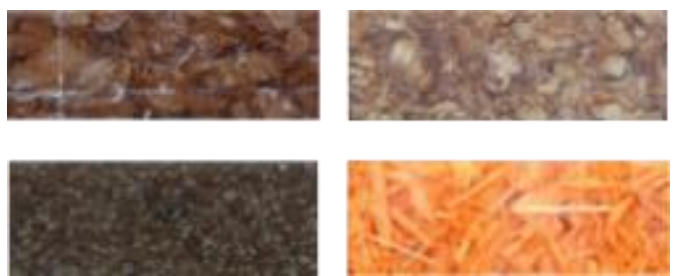

Figure 3. Macro Structure of wood flour : very course, course, fine/medium and length dimension 
The dimensions of wood flour as the filler are also investigating both a square shape and the shape resembles a short fibre length. 2 types of wood flour (semaram and cengai) was observed as hown in Figure 4.

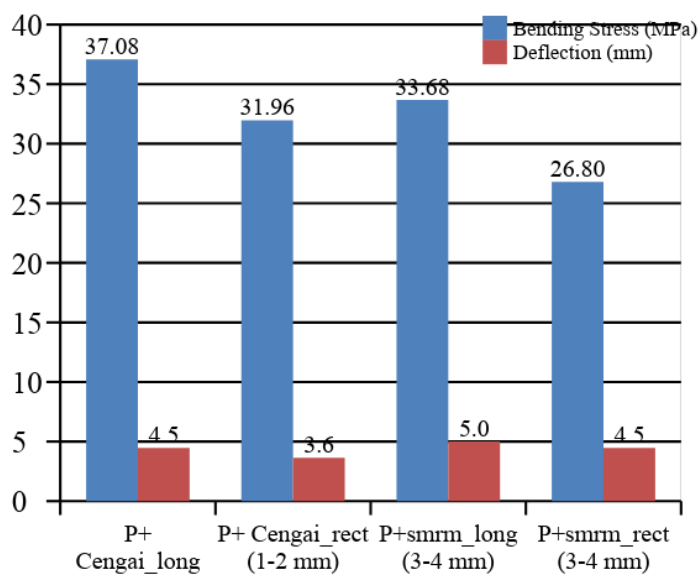

Figure 4. The influence of the dimensions of the wood flour filler of cengai and seumaram (long and square) on the bending strength of the wood flour polyester composite materials

The shape/dimension of wood flour as filler has an influence on the strength of wood flour polyester composite materials. Base on the figure 5, filler in long dimension resemble to fiber is better bending strength than the rectangle one. The failure mechanism was initiated from the micro cracks and will propagate due to external forces that loading up the material to failure. The presence of wood flour as filler in length shape was possible to become an obstacle to crack propagation, or can change the direction of crack propagation which is possible to delay of failure that make the material stronger, as shown by Figure 5 .
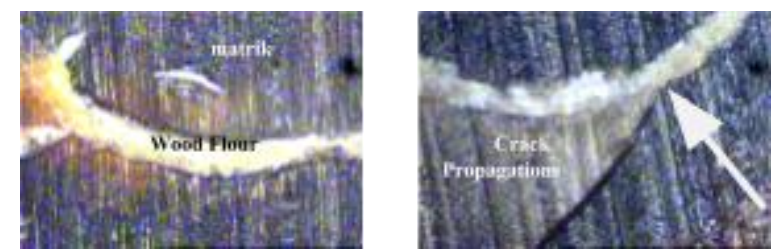

Figure 5. Macrostructure of length wood flour filler (resemble as wood fibre) could inhibit the direction of crack propagation

On the other hand, the fine of wood flour filler in form of rectangular dimension, the bending strength is slightly lower due to dimension effect.

\subsection{The influence of the type of wood flour Filler}

There are 5 types of wood flour filler were investigated such as: wood flour filler of Jeumpa, Meuranti, Damasui, Seumaram and Cengai. The three kinds the fist wood flour are classified as softwood, while the latter two types are classified as hardwood. Figure 6 shows 
the influence of the type of the rough wood flour on the bending strength of the wood flour polyester composite materials.

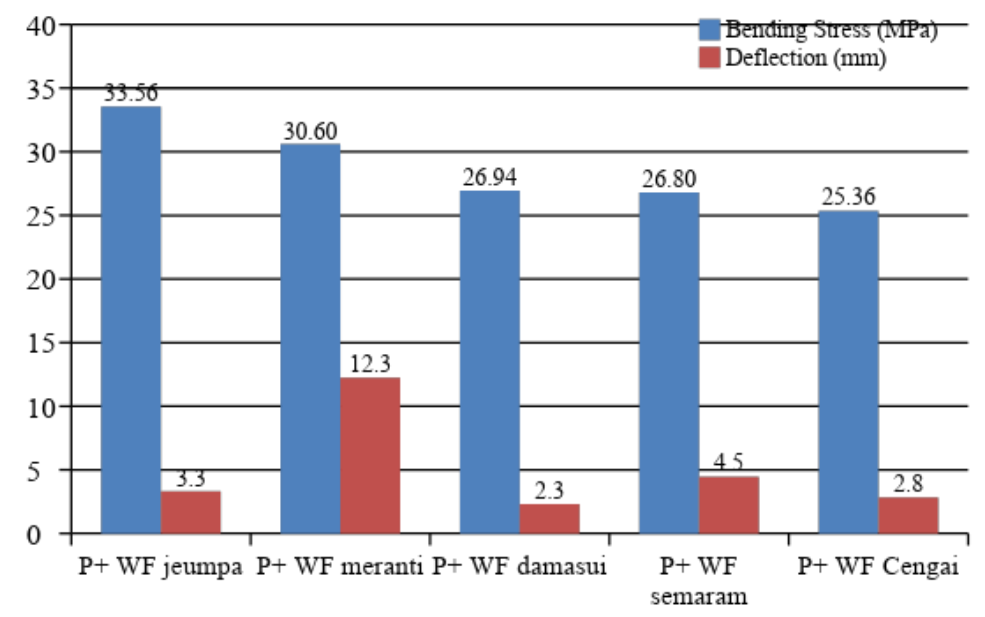

Figure 6. Effect of type of wood flour filler on the bending strength of wood flour polyester composite materials

Figure 6 explains that the wood flour of Jeumpa has the highest bending strength, followed by Meranti, Damasui, Seumaram and cengai. This indicates that the kind of hard wood filler tend to have lower bending strength than the soft wood filler. This phenomenon is closely linked to the condition of the bond formed between wood flour filler and the polyester resin matrix. The good bonding will establish when the polyester resin can enter seep into the wood flour to form 'cross linking'. The ability of softwood filler in absorbing resin (wettability) looks good enough to produce a bond which is quite compact on the interface, while coarse sawdust filler looks less solid at the interface as shown in Figure 7.
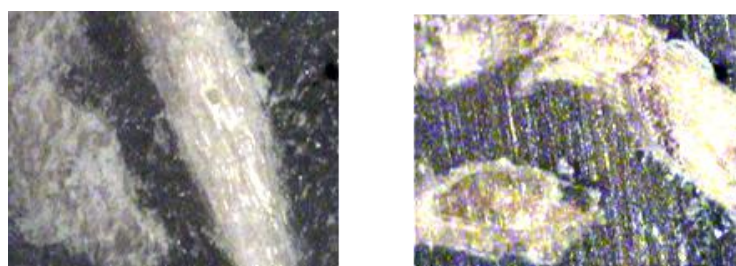

Figure 7. Macro Structure of the Jeumpa wood flour filler shown more cohesive at interface and the Cengai wood flour shown less coherent within the polyester matrix.

The wood flour fillers of Cengai types showed a cavity in the interface between wood flour filler and polyester matrix that possible to becomes a source of crack initiation as a trigger for the specimen failure. On the other hand, the soft wood flour filler shows the compact bonding at the interface. 


\subsection{Influence of sandwich with the fibre glass to bending strength of wood flour polyester composite.}

The wood flour Polyester composite material has a low strength so it cannot be applied to the engineering structural materials. By reinforcing with the glass fiber on both surfaces to form a composite sandwich, it was expected to be more robust and suitable for structural applications. The influence of reinforcement using fiberglass on both surfaces of the wood flour polyester composite are shown in Figure 8. It explains that the sandwich with polyester of fiber glass (as skin) can improve the strength of the wood flour polyester composite (as core) up to $\pm 45 \%$ so that the material has good prospects for structural applications. It was resulted from a good combination of both that produce the good bonding at the interface.

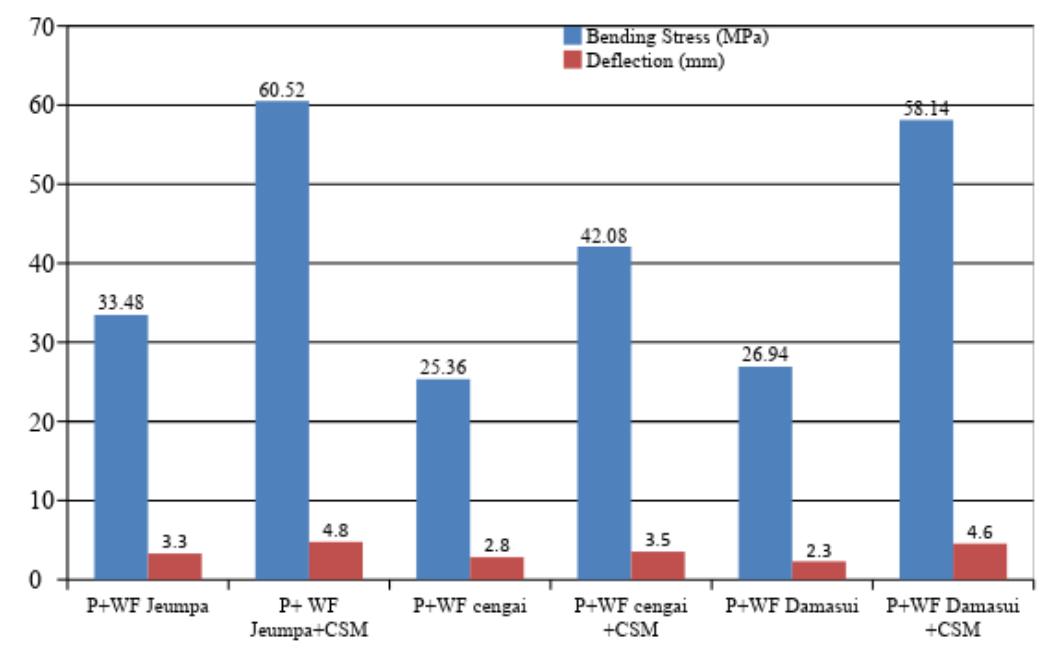

Figures 8. Effect of CSM fiber glass reinforcement on the bending strength of the wood flour polyester composite

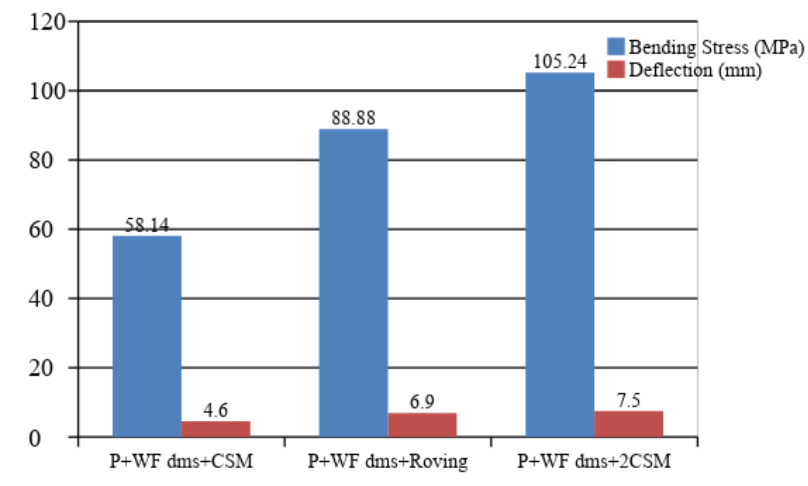

Gambar 9. Effect of type of fibre glass reinforcing (CSM and Roving) on the bending strength of the wood flour polyester composite material 
The fiberglass plays an important role in the process of making the sandwich composite materials between the wood flour composite and fiberglass composite. There are two types of fiberglass used widely by the community; it was Chopped Strant Mat (CSM) and the type of fiber roving. The effect of strengthening that produced by these two types of fibers to the wood flour polymer composite was very different, as shown in Figure 11. It can be shown that the reinforcement with Roving fiberglass provide a greater effect than the CSM fiber glass. However, the two layers of CSM fiberglass provides an increase in strength is proportional to 1 layer of roving type of fiberglass. This indicates that the density of the fibers has a considerable influence on the reinforcing effect produced.

\section{Conclusion}

The alkaline and borax treatment for the wood flour and the addition of coupling agent MAH did not provide a positive influence on the strength of the wood flour polyester composite materials. The size and dimensions of wood flour filler have the influence on the bending strength, in which a medium-size $( \pm 2 \mathrm{~mm})$ and the length dimensions can provide a good reinforcement effect. While the type of soft wood flour filler can produce a better bending strength than the hardwood filler. Furthermore, the process of sandwiches using 1 layer of fiberglass can increase the strength of the wood flour polyester composite materials up to $45 \%$, where the type of woven fiberglass (Roving) has a reinforcing effect that up to 45 $\%$ better than fiberglass type CSM. However, when CSM fiber in use two layers, the effect is almost the same gains with 1 layer of fiberglass Roving type.

\section{References}

[1] Debiprasad Gon, Kousik Das, Palash Paul, Subhankar Maity. Jute Composites as Wood Substitute, International Journal of Textile Science, (2012).

[2] Stark, N. M. Wood Handbook, Chapter 11 :Wood Based Composite Material Panel Product-Glued Laminated Timber, Structural Composite, Forest Products Laboratory USDA Forest Service Madison, Wisconsin, (2010).

[3] Camelia Cerbu 2015, Practical solution for improving the mechanical behaviour of the composite materials reinforced with flax woven fabric, Advances in Mechanical Engineering,DOI:10.1177/1687814015582084, (2015).

[4] Sanjay, K. M. Composites Manufacturing : Materials,Product, and Process Engineering, CRC Press, (2002).

[5] Camelia Cerbu, Ioan Curtu, Vasile Ciofoaia. Effects of the Wood Species on the Mechanical Characteristics in Case of Some E-glass Fibers/Wood Flour/Polyester Composite Materials, MATERIALE PLASTICE 47 Nr. 1, (2010). 